\title{
Monitoring of Indian Agriculture using LPC2148
}

\author{
S. Lakshmana Pandian \\ Associate Professor /CSE \\ Pondicherry Engineering College
}

\author{
Y. Charishma \\ B. Tech,CSE \\ Pondicherry Engineering College
}

\author{
V Parameswary \\ B. Tech ,CSE \\ Pondicherry Engineering College
}

\begin{abstract}
In this paper, we describe about the monitoring of Indian agriculture using LPC2148.Monitoring of Indian agriculture is done using information of the temperature and humidity content. This is mainly used for saving water and monitoring agriculture without human presence. Temperature sensor and humidity sensor will continuously sense the information regarding the field. When the values are less than or greater than the threshold values it will do certain operations. We are using GSM technology also for sending information to the farmer. We have included two modes for monitoring manual and automatic mode. In manual mode farmer will be sending message and to monitor and control the water pump. In automatic mode farmer will not be involved to control the operations of motor .It will be operating the water pump motor automatically. So, that he can monitor from remote places also. As ARM7 processor is RISC architecture, it is so flexible to program and as advancements has increased in this field we have done code optimization for the program in this project.
\end{abstract}

\section{Keywords}

LPC2148,LM35,GSM,code optimization, algorithm

\section{INTRODUCTION}

In this paper we mainly discuss about monitoring of agriculture field without the presence of the human in the field. We require monitoring of agriculture these days because in this modern world as the technology has taken tremendous changes in many fields we are going to implement in the field of agriculture. As the time has playing key role we have introduced this system so that farmer can save some time and need not go to farm everytime. Now a days resources are getting drained so we should not waste them and use the existing resources carefully. So in order to avoid the wastage of water we have made an automatic control of water pump motor. So this project will help in protecting resources and saving time. In this project we does need a desktop to see the status of the farm. We have a LCD display to see the status of the farm and GSM modem is used to send message to farmer regarding the status of the farm. Farmer can know the information of the farm from the remote places also..For this project we have taken the LPC2148 microcontroller because it is a 64-bit RISC architecture. So that is can be easy to write the program with less instruction set and if we want to add any advancements we can add easily to them. We can have many other sensors required for farm to have advancements in the project. As the advancements regarding monitoring of agriculture using ARM7 are increasing we have tried for code optimization technique. So that by doing code optimization we can decrease the time and space complexity.

\section{PROPOSED SYSTEM}

In this proposed system, the sensors will continuously sense the data and sends to microcontroller. The information regarding temperature and humidity received by the microcontroller is displayed on LCD display in the farm. When the data received is minimum or maximum of the threshold value it will take particular actions depending on the value. The water level sensor senses the level of the water. When the water level is low it will send the message to the farmer that the water level is low using GSM. Then the farmer sends message(AT commands) to $\mathrm{ON}$ the motor. When the water reaches the maximum then water will turn off automatically. So that we can avoid wastage of water. We will be giving date and time when we start the system, so that during night time the light in the farm will automatically $\mathrm{ON}$ and when in the morning the light will automatically OFF. The date and time will also be displayed on the LCD screen. We are going to do the code optimization so that we can reduce the time and space complexity.

\section{DESIGN OF THE SYSTEM:}

The design of the system involves both hardware and software. The hardware of the system will be placed in the farm and we require software for programming the hardware and loading the program in to flash memory.

\subsection{Hardware Requirements:}

The hardware of the system contains the

- $\quad$ LPC2148(ARM microcontroller)

- $\operatorname{GSM}($ Global System for Mobile communication)

- LM35(Temperature sensor)

- Humidity Sensor

- Water level sensor

- Switch

- Relay circuit

\subsubsection{LPC2148}

ARM7 is one of the widely used micro-controller family in embedded system application. ARM is a family of instruction set architectures for computer processors based on a reduced instruction set computing (RISC) architecture. A RISC-based computer design approach means ARM processors require significantly fewer transistors than typical processors in average computers. This approach reduces costs, heat and power use. The LPC 2148 incorporates a 512 KB Flash memory system. This memory may be used for both code and data storage. The flash memory can be programmed using serial built-in JTAG interface, using In System Programming and UART0 or by means of In Application Programming capabilities. The application program, using the IAP functions, may also erase and/or program the Flash while the application is running, allowing a great degree of flexibility for data storage field firmware upgrades, etc. When the LPC2148 on-chip boot loader is used, $500 \mathrm{~KB}$ of Flash memory is available for user code.

The LPC2148 Flash memory provides minimum of 100,000 erase/write cycles and 20 years of data-retention. The LPC2148 has two ports $\mathrm{p} 0$ and $\mathrm{p} 1$. It has 64 pins where each port has 32 pins. The ports are represented as PXY where $\mathrm{X}$ represents the port number and $Y$ represents the pin number 0 to 31 . Each pin is having a feature of performing the alternate function. ON 
RESET, each pin is configured as GPIO. For any of the other use programmer must configure it properly.

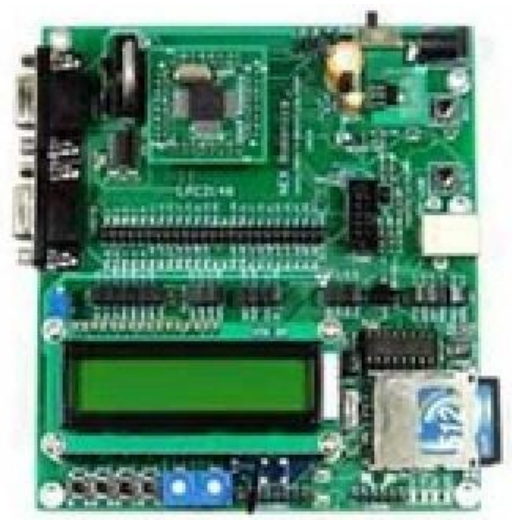

Fig 1. LPC2148

\subsubsection{GSM}

Global System for Mobile was the world's first cellular standards. It is a digital mobile telephony system.GSM services follow ISDN guidelines and are classified as either tele services or data services. The remarkable feature of GSM is the Subscriber Identity Module(SIM), which is a memory device that stores information such as the subscriber's identification number.GSM digitizes and compress data, then sends it down a channel with two other streams of user data, each in its own time slot. It operates at either the $900 \mathrm{MHz}$ or $1800 \mathrm{MHz}$ frequency band.

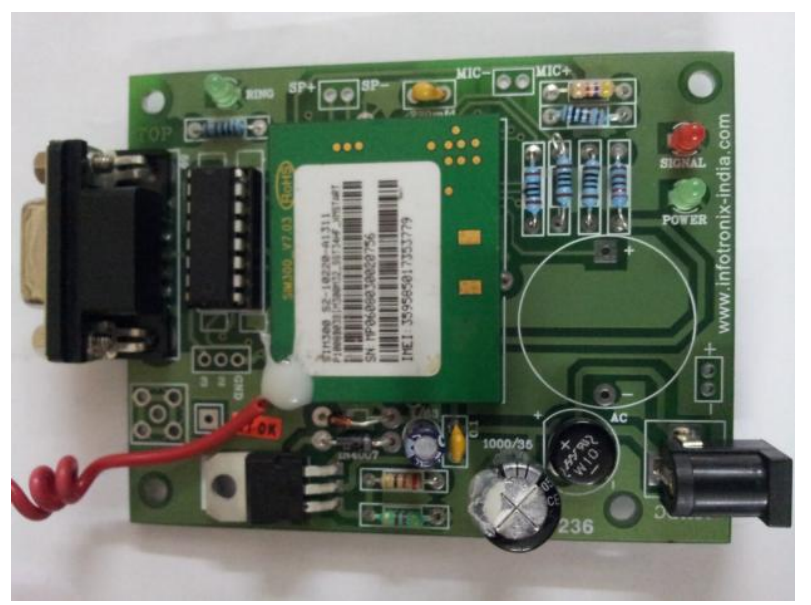

Fig 2. GSM

\subsubsection{LCD Display}

LCD(Liquid Crystal Display) screen is an electronic display module and find a range of applications. A $16^{*} 2$ display is a basic module and commonly used in various devices and circuits. These are seven segments and other multi segment LEDs. It has two registers command and data each.

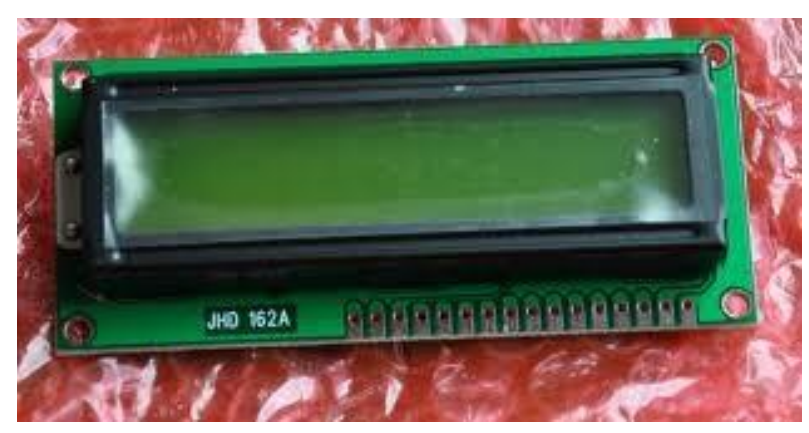

Fig 3. LCD Display

\subsubsection{Humidity Sensor}

Humidity is the presence of water in air. The amount of water vapor in air can affect human comfort as well as many manufacturing process in industries. In this project we have used hrt393 humidity senosr for sensing the humidity content and displaying the humidity in LCD screen.

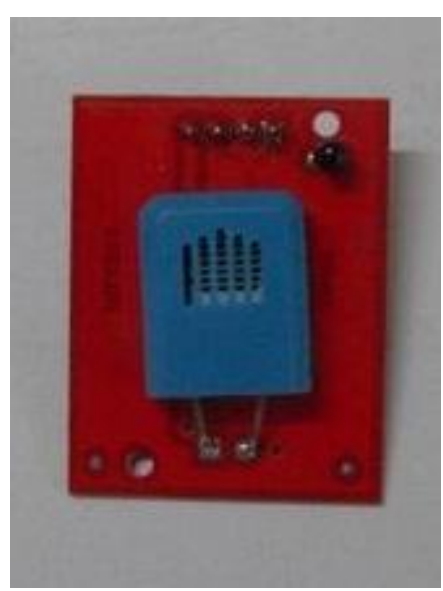

Fig 4. Humidity sensor

\subsubsection{Temperature Sensor}

The LM35 series are precision integrated-circuit temperature sensors, whose output voltage is linearly proportional to the Celsius (Centigrade) temperature. The output of sensor converted to digital that easy connecting with microcontroller.

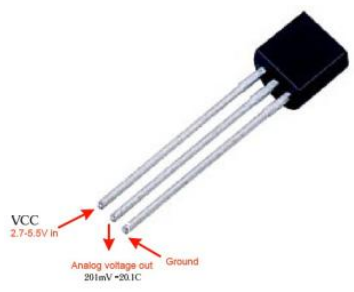

Fig 5. LM35 


\subsubsection{Water Level Sensor}

Water level sensor in this project is made of resistors for getting the information of the level of the water. It has two conditions low and high. When the water level is low it will send the information to ARM microcontroller and when it reaches high it will send the information to the controller.

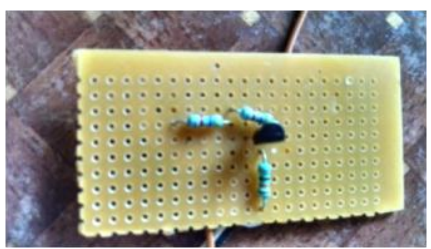

Fig 6. Water level sensor

\subsubsection{Switch}

Switch is used for increasing the voltage. Because water pump motor requires $12 \mathrm{~V}$, and ARM7 board and GSM requires different voltages. So to control the flow of voltage we have used Switch

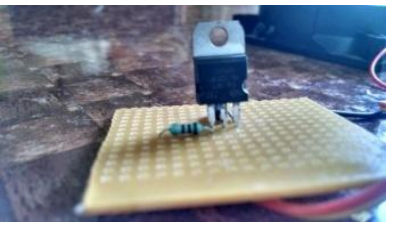

Fig 7. Switch

\subsection{Software Requirements:}

We need software for programming the device and make it run according to our needs.

\subsubsection{Keil $\mu$ vision IDE}

Keil $\mu$ Vision is a free software which is mainly used for Embedded Systems. This Keil $\mu$ Vision is an integrated development environment (IDE), which combines the text editor/notepad for writing C language codes or Assembly language codes and a compiler which converts the source code to hexadecimal codes. We can also test the program without the hardware.

\subsubsection{Flash Utility}

For loading the program into the controller, we are using Philips Flash Utility Tool to load the hex codes to the ARM controller through UART port so that we cannot compile the code every time when we are running.

\section{ARCHITECTURE}

The water level sensor ,soil moisture sensor, temperature, humidity sensor are connected to the ARM microcontroller. The temperature /humidity measured by the sensor is given to the farmer using GSM. We are using MAX232 for connecting the microcontroller and GSM. With the information coming from the soil moisture sensor it will make the motor pump automatically ON/OFF. When the water level reaches the adequate level, water level sensor will send the information to the microcontroller and makes the water pump motor OFF. If the farmer wants to know the status of farm then farmer can request through GSM. The current status will be sent to the farmer. We have used Relay circuit to represent lamp which will automatically on/off according to the time given by the user. So we have used the real time clock to monitor this project.

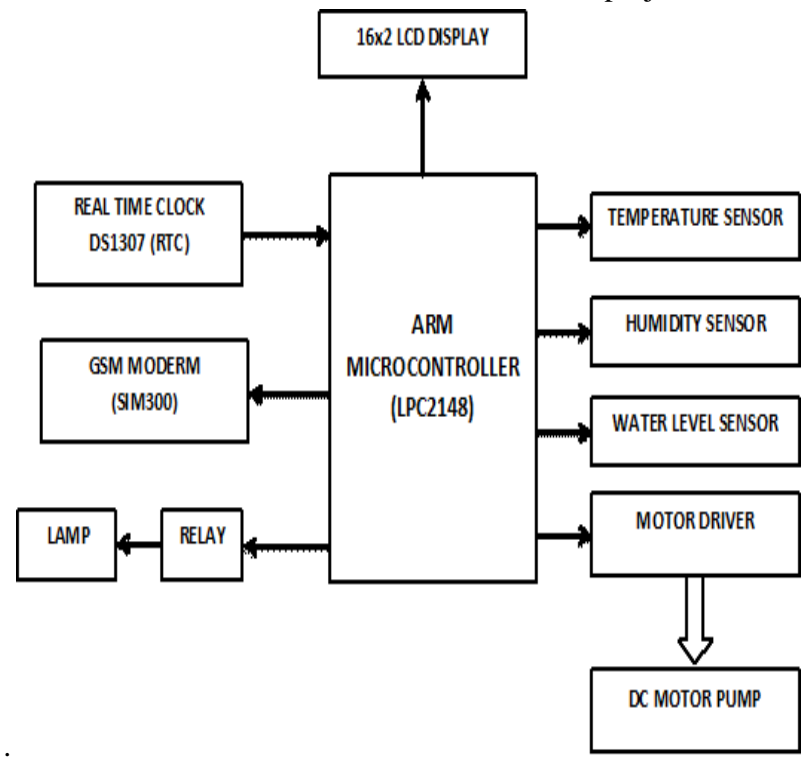

Fig 8. Architecture

\section{IMPLEMENTATION}

Initially power is giving continuously to the system so that the relay distribute the power to the respected devices in the system. After this, GSM is initialized by getting signal with delay. We have used a keyboard to give the input for microcontroller like date and time according to which the lamp will work. When we switch $\mathrm{ON}$ the system Temperature level, Humidity level, Water level and time will be displayed on the LCD screen. Depending upon the time, the lamp is $\mathrm{ON}$ in the night time and it will be OFF automatically in the day time. If the water level is LOW in the field, the farmer will get the message as water level is low including temperature level and humidity level and command to $\mathrm{ON}$ the motor. Once the farmer sends AT command as \#ON then the motor will be ON. If the water reaches the maximum level, automatically motor will be OFF.

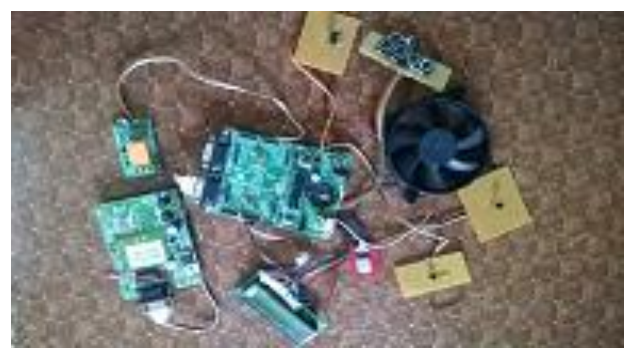

Fig 9.complete system for monitoring

In the above figure we can see the complete system that can be placed in the form. We have interfaced temperature, humidity and water level sensors. We placed GSM and LCD display for monitoring. We have small keypad to give the inputs.

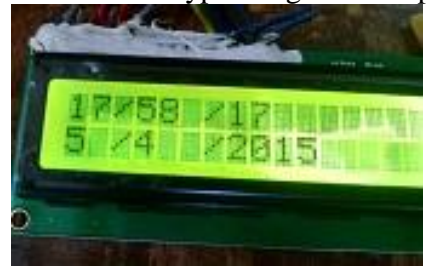

Fig 10. Displays time and date 
The user will be giving the inputs using the keypad, where real time clock is used here. The time and date will be displayed on the LCD display.

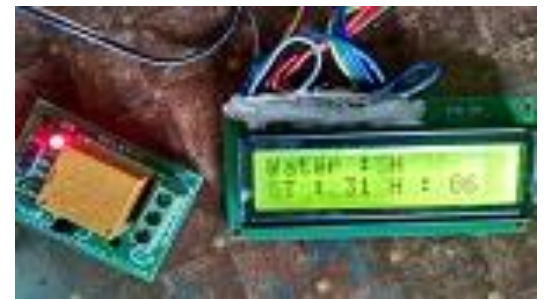

Fig 11. Displaying the water and humidity levels

When We start the system then sensors will sense the data and automatically send the information to the microcontroller. It will display the data in the LCD display regarding the water level ,temperature and humidity level.

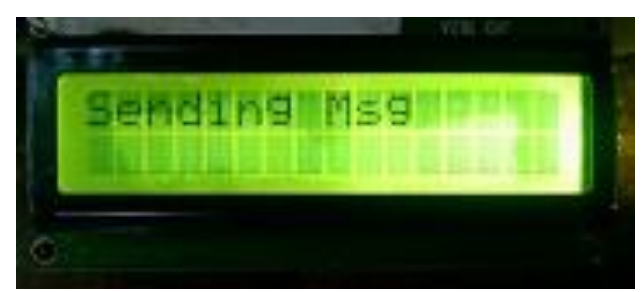

Fig12 Sending msg when water level is low

When the water level is LOW then water level sensor will send the information to the microcontroller. The microcontroller will send the information to the farmer using GSM. The sending of message is displayed on the LCD display.

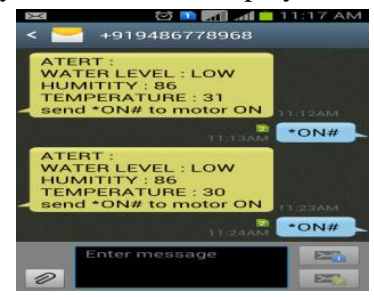

Fig 13. Farmer receiving messages and sending the AT command

The farmer will receive the message regarding the status of the farm when the water level is LOW. Then farmer sends an AT command $* \mathrm{ON} \#$ command to $\mathrm{ON}$ the motor.

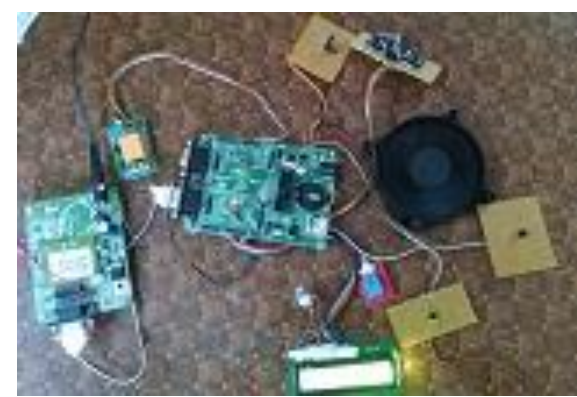

Fig 14. Motor ON

When the farmer sends an AT command then GSM will send that information to the microcontroller and water pump motor will $\mathrm{ON}$ automatically.

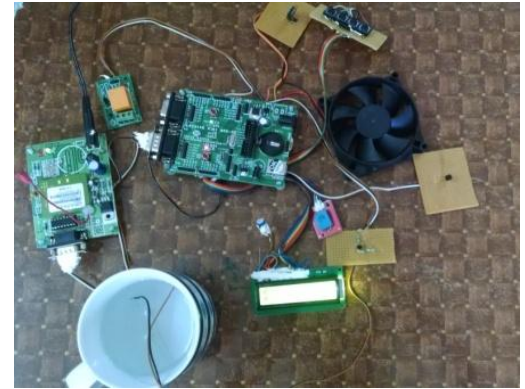

Fig 15. Motor OFF

When the water level reaches HIGH then motor will OFF automatically.

\section{FUTURE SCOPE}

- We can monitor the field through camera and can send as MMS to the farmer.

- We can find the disease of the plant by placing the sensor at roots of the plants and can give suggestions regarding the fertilizers to the farmer.

\section{CONCLUSION}

In this project we are monitoring the farm using few sensors The status of the farm will be known to the farmer from remote place also. We are controlling the water pump motor from remote place. So we are reducing the human labour power and resources where we can prevent the wastage of resources

Regarding the microcontroller we are loading the program into flash memory, where it will retain for atleast 20 years. We can do changes to the program easily due to is its instruction set..So ARM7 microcontroller is more efficient when compared to other microcontrollers.

\section{REFERENCES}

[1] Joaquín Gutiérrez, Juan Francisco Villa-Medina, Alejandra Nieto-Garibay, and Miguel Ángel Porta-Gándara, "Automated Irrigation System Using a Wireless Sensor Network and GPRS Module", in Proceedings of the IEEE transactions on instrumentation and measurement, vol. 63 , 166, January, 2014.

[2] M.Ramu and CH.Rajendra prasad, "Cost effective atomization of Indian agricultural system using 8051 microcontroller", International Journal of Advanced Research in Computer and Communication Engineering, vol. 7, Issue 7, July, 2013.

[3] Prathyusha.K, G. Sowmya Bala and Dr. K. Sreenivasa Ravi, "A real - time irrigation control system for precision agriculture using WSN in Indian agricultural sectors", International Journal of Computer Science, Engineering and Applications (IJCSEA), vol. 3, August, 2013.

[4] Gayatri Londhe, Prof. S.G. Galande, "Automated Irrigation System By Using ARM Processor", International Journal of Scientific Research Engineering \& Technology (IJSRET), vol. 3, 254, May, 2014.

[5] G. V. Satyanarayana and SD.Mazaruddin, "Wireless Sensor Based Remote Monitoring System for Agriculture Using ZigBee and GPS", in Proceedings of the Conference on Advances in Communication and Control Systems 2013 (CAC2S 2013), 2014

[6] K. Chenna Kesava Reddy and P.Venkatrao, "Real Time Field Monitoring and Controlling System", International Journal of Recent Technology and Engineering (IJRTE), vol. 2,. September, 2013 
[7] Chandrika Chanda, Surbhi Agarwal and Er. B.Persis Urbana Ivy, "A Survey of Automated GSM Based Irrigation Systems", International Journal of Emerging Technology and Advanced Engineering, vol. 2, October, 2012.

[8] Ms. Shweta S. Patil and Prof. Mrs. A. V. Malviya, "Review for ARM based Agricultural Field Monitoring System", International Journal of Scientific and Research Publications, vol. 4, February, 2014. 50

[9] Karthik Maddipatla, Thentu Sravani, Thota Rajesh, R.S.V. Mani Krishna and J.Avinash "Remote Access to Agricultural Motor through the Usage of GSM and SMS Technologies" vol.1, 2012.
[10] Purnima and S. R. N. Reddy "Design of Remote Monitoring and Control System with Automatic Irrigation System Using GSM - Bluetooth” on IJCA, 2012.

[11] Mahesh M. and Galgalikar, "Real-Time Automization Of Agricultural Environment for Social Modernization of Indian Agricultural System", on IEEE Proceedings, 2010.

[12] M. Nagendra Babu and Indira Priyadarshini S. "Real Time Automation of Indian Agricultural System".

[13] Vasif Ahmed, Siddharth A. and Ladhake, "Design of Ultra Low Cost Cell Phone Based Embedded System for Irrigation", on International Conference on Machine Vision and Human Machine Interface, 2010. 